\title{
Material Identification
}

National Cancer Institute

\section{Source}

National Cancer Institute. Material Identification. NCI Thesaurus. Code C138993.

Tests that establishes the characteristic and uniqueness of the substance of interest and should be able to discriminate between compounds of closely related structures which are likely to be present. 